tube, being healthy, were not removed. The patient made an uninterrupted recovery.

CASE V.-T. L., aged 25, married seven years, had three children, the last born three years ago ; all the labours were difficult; she had prolapse of the womb after the second. Since the last confinement, the uterus had not been prolapsed, but she had suffered from violent "bearing-down" pains, had had painful menstruation, and had been unfit for active employment. The uterus measured three and a half inches, and behind it was a tumour doubtfully fluctuant. On November 27 th, abdominal section was performed. The omentum was adherent over the pelvic brim. When pierced by the fingers, dark fluid blood welled up, and was mopped with sponges. Both tubes were greatly hypertrophied, and filled with old dense bloodclot; both fimbriated extremities were expanded and inflamed, the right one being partly spread over a dense clot of the size of a goose's egg. The ovaries were atrophied: The cæcum and appendix were drawn down into the pelvic cavity, and their adhesions to the right tube proved somewhat embarrassing. The left tube appeared to have insinuated itself behind the rectum, and was with difficulty detached. After removal of both uterine appendages, a small rupture of uterine tissue, near the cervical junction, was discovered, and there was considerable bleeding in Douglas's space. The abdominal incision was enlarged from one inch and a half (its ordinary length) to three inches, so as to cnable me to stitch the uterine tear, and to ligature several bleeding points deep in the pelvic cavity. The peritoneum was raised from the posterior portion of the left broal ligament, like the epidermis of a blister, but it did not bleed. A long glass drainage-tube was used for two days, and then a short one for twenty-four hours. During the operation, a quantity of blood flowed into the vagina, which was probably due to the squeezing of the tubes during their withdrawal. The patient has made an excellent recovery. She relished her soup on the second day, and, though she has not yet (December 5th) been allowed to rise, the pulse and temperature have been normal for many days, the incision has practically healed, and she has lost all pain.

REMARKs. - In none of these cases was there evidence of tubal pregnancy. The extreme collapse, which is, perhaps, the most significant symptom of rupture in this condition, was never noticed. It is, of course, possible that a small disintegrated foetus, or a corpus luteum, may have been a pathological factor in all of them. There was also no history of illness commencing with an abrupt cessation of the menses, a symptom so often alluded to in text-books. In all except the fourth case, there had been long enduring menorrhagia. Bernutzand Goupil(Diseases of Women, Sydenham Society, vol. i, pp. 180-188) have narrated, from their personal experience, five cases remarkably similar to mine, occurring in women between the ages of twenty-one and twentyeight. In all of them, "death took place either suddenly or after a short illness;" but the symptoms and the post mortem examinations showed that peritonitis, and not internal hæmorrhage, was the immediate cause of death. The subsequent history of my five cases, I may say of my six cases, shall be faithfully reported.

\section{A CASE OF ACUTE MENINGITIS.}

Read before the Aberdeen, Banff, and Kincardine Branch.

BY FRANK OGSTON, M.D.,

Medical Examiner in Criminal Cases for the City and County of Aberdeen.

A CHILD nearly 9 years of age, who had been dull and listless for a fow days, and who, in consequence, had been kept from school, when roused one morning, complained bitterly of pain in the head and sickness. She was accordingly left in bed, and, falling asleep, slept till two o'clock in the afternoon. On awaking, she felt better, and took some food. The next day, she seemed well, and got out of bed; after which, she passed a good night. On the third day, however, she again complained of her head, and said that her eyes were sore. She took tea, but no food, and remained in bed. At three in the afternoon, she had some difficulty in breathing; "her throat was closed," as her mother said. A poultice was applied to it, and her mother took her out of bed, and sat with her upon her knee till half-past five o'clock, when she died. No medical man was called in.

I made a post mortem examination of the child two and a half days after death, finding the following appearances.

Externally. - There were no noticeable signs of neglect or appearance of want of ford. The joints were rigid. The pupils of the eyes were slightly dilated, the left more so than the right. The lips were dry. The finger-nails were white. The front of the body was pale the back parts purplish. There was nothing else unusual observable on the exterior of the body.
Internally. - The scalp was pale, and the bones of the vault of the skull were normal. On removing the skull-cap, the dura mater appeared healthy. When the brain was exposed to view, the whole of its upper surface was of a pale yellow colour, with blood-vessels partly distended with, partly empty of, blood, ramifying across its surface and, when the arachnoid was cut into, a gelatinous semifluid substance oozed from underneath it. This substance was found to occupy the subarachnoid space alone; for, when the pia mater was also cur through, it stripped readily from the brain, and showed its surface healthy, to the naked eye at least; the only thing that was remarked being that the convolutions appeared a little crowded together, but this was doubtful. This morbid appearance was confined to the meninges of the superior surface of the brain, with the exception of a little tongue-like extension on that part which lay to the inner side of the orbital plate of the ethmoid bone. Otherwise, the basal meninges were quite healthy. The brain was now removed from the skull, and the dura mater carefully examined; but it was found healthy, except a small patch which covered the orbit, which was thickened, roughened on the side next the bone, and covered with a layer of lymph on the side next the brain, by which it was glued to the arachnoid; anı also at the parts overlying the middle ears, where it was roughened and thickened on its outer side. The orbital plate of the right side presented a canary-yellow appearance, and a congested and roughenel state of the bone. On cutting this through, the nasal cavity was found to be filled with thick creany pus, which welled out into the brain-cavity. The middle ears were then opened from above, and were found to be filled with dirty-reddish fluid. 'The mouth, throat, gullet, and air-passages were natural. The heart and its valves were healthy; its central cavities contained blood partly fluid, partly as dark clots, and a large fibrinous clot was found in the right ventricle. 'The lungs contained a moderate quantity of blood, mostly in their posterior parts, and were attached in a few places to the chest-walls by old pleuritic adhesions. The liver, spleen, and kidneys were congested; the liver and kidneys having the appearance of slight amyloid degeneration, and the spleen being considerably enlarged and very soft. The stomach contained merely a little dirty grey fluid. 'T'he sigmoil flexure of the large intestine was distended with gas, the rest of the bowels, in contrast to it, being flat and collapsed. Nothing else unusual was observable in the interior of the body.

Here we have a case of simple acute meningitis, a disease rart enough in itself to be interesting; but this particular case is noteworthy from the fact that the cause of it could be easily traced.

This exciting cause was, it is evident, inflammation of a chronic nature probably, of the nasal mucous membrane, which had sprearl through the orbital plate of the ethmoid bone and the dura mater to the arachnoid membrane, which it had attacked, and the inflammation had spread along the subarachnoid space.

The history of the illness, imperfect though it be, is typical of this disease, namely, pain in the head and sickness, followed by intolerance of light (complaint of sore eyes), and the short duration of the illness.

But what makes this case still more interesting is, that another child of the same family was attacked by, and died a few days afterwards from, a disease which the medical man who was called in at onc. diagnosed as meningitis, and that the father fell ill with something of the same nature.

\section{NASAL ASTHMA : ITS CAUSES AND TREATMENT.}

BY G. HUNTER MACKENZIE, M.D.,

Surgeon for Throat-Diseases, to the Eye, Ear, and Throat Infirmary, and to the Western Dispensary, Edinburgh.

THE causal relationship between nasal disease and asthua was first pointed out by Voltolini, in 1872 (referred to by Morell Mackenzie, Manual of Diseases of the Throat and Nose, vol, ii, p. 360), and since that time numerous cases have been recorded which have established the accuracy of this observation. In all these instances, with one exception recorded by myself (Edinburgh Medical Journal, February, 1883), polypi, or marked swellings of the nasal mucous membrane, have been the varieties of disease present. This has given rise to the theory held by some, that the asthma is owing to mechanical obstruction to the passage of air; and additional confirmation of this view is supposed to be afforded by the disease disappearing on the removial of the obstructing mass.

Against this theory two arguments can be advanced; the frequency of polypus or tumefaction of the nasal mucous membrane without asthma, and the presence of nasal asthma without the occurrence of either. The first is a matter of every-day experience, and the second has received illustration by the case above referred to, in which violent 
paroxysms of asthma were associated with a condition of chronic (atrophic) inflammation of the nasal mucous membrane, and ceased on the application of nasal remedies; and by the following case that has recently came under my notice.

A boy, aged 13, was brought to me on January 23rd, 1885, on account of a copious watery discharge from the nose, and asthma. He had suffered from these for about ten years, with slight periods of remission. The asthmatic attacks were often very severe, and generally occurred about 4 or 5 A.M. The condition of his nose necessitated the use of from twenty to thirty handkerchiefs daily. Anterior and posterior rhinoscopy showed chronic eatarrh of the nasal mucous membrane, with a slight amount of muco-purulent secretion. There was no polypus or thickening of the membrane. Though not robust, he presented no indications of lisease elsewhere. (This patient has, apparently, quite recovered, under the after-mentioned treatment. )

What is the explanation of such cases? I believe the asthma to be owing, not to any mechanical obstruction of the nasal passages, but to a condition of abnormal irritability of the nasal mucous membrane, lue to, or aggravated by, chronic inflammation. Polypi may, or may not, co-exist; when present, they doubtless assist in maintaining the augmented irritability of the mucous lining. The high degree of normal sensitiveness of the mucous membrane of the nose is well known; and when this membrane has been the subject of long-continued inflammation, its irritalility appears to become highly augmented, and more easily excited. Reflex acts are then readily induced, of which sneezing, cough, and asthma are the principal indications.

The peripheral irritation may be caused by dust or pollen, and hence the attacks are usually worse in midsummer and autumn; by cold, therefore their frequency in the early morning hours; or by the irritation of a polypus. Dr. John Mackenzie has recently stated (Transactions of the Medical and Chirurgical Faculty of Maryland, 1884) that asthmatic attacks in cases of nasal polypus only occur when the growth is, by force of gravity; brought against the posterior part of the nostril, corresponding with the most excitable spot in the sensitive area. On the other hand, Hack, quoted by Morell Mackenzie (Manual of Diseases of the Throat and Nose, vol. ii, p. 361), considers that reflex phenomena may be produced by irritation of any part of the lining membrane of the nose.

Associated with the asthma are, usually, excessive sneezing, and profuse discharge of mucus from the nose, occasionally cough. There is more or less impairment of the general health, with languor, and depression of spirits.

The treatment of these cases must obviously, in the first place, be directed to the removal of the exciting cause. The patient ought to be directed to reside in an atmosphere as free as possible from dust and other atmospheric impuritics. Above all, active medication should be directed to the nose; and, after a fair trial of various remedies, I have found none so efficacious as belladonna, applied in the form of buginaria (nasal bougies). From one-twefth to one-sixth of a grain of the extract ought to be incorporated in each bougie, one of these being introduced into each nostril night and morning, and allowed to thoroughly dissolve there. I have found their employment most beneficial, alike in regard to the sneezing, the secretion, and the asthma. Their use is usually followed by a burning sensation in the nose, but this is never severe, and soon disappears. I have also experimented with the sulphate of atropia in the same way, but have found it less efficacious, and probably nore irritating than the extract. Previously to the introduction of the bougie, the nasal mucous membrane may be cleaned by the anterior nasal spray. The use of the bougies may be gradually made less frequent, according to the amount of success achieved.

The cases which I have hitherto watched, have rather belonged to the atrophic than the hypertrophic form of rhinitis, but where vascular engorgement is present, it seems probable that the use of cucaine bougies would be beneficial. According to Bosworth (Internationales Centralblatt für Laryngologic und Rhinologie, No. 8), this drug has a remarkable effect in diminishing the vascularity of the lining membrane of the nose. In acute nasal catarrh,its local application has only a temporary effect.

The treatment may be combined with the administration of tonics, and such other general remedies as may be deemed suitable by the physician. By themselves, these are, however, singularly inefficacious. I venture, therefore, to commend to the profession the use of nasal bougies, containing belladonna, as an effectual method of treating the troublesome and obstinate complaints dependent upon chronic nasal catarrh and irritability, of which asthma is alike the most im. portant and most intractable.

\section{NOTE ON THE USE OF HARROGATE WATERS. \\ By JAMES A. MYRTLE, M.B., C.M., Harrogate.}

In December, Dr. Eddowes, of Shrewsbury, wrote, saying he had a most obstinate case of eczema, and asked how soon the patient might be sent to Harrogate for the baths and waters. I answered, any time, as the action of the waters in such cases was quite as beneficial in the winter as in the summer.

On January 5th, Mrs. J., the patient, arrived. I have only seen one worse case of impetiginous eczema, which was in the skin-wards of the Allgemeine Krankenliaus in Vienna. Mrs. J.'s heal, face, neck, breast, arms from the elbow-joints, and hands, were a mass of yellow crusts, with a putrid, but not favus-like, smell. No favus-microbe was discovered. The patient's liver and organs of digestion were completely clogged; the urine was high-coloured, and very small in yuantity. A calomel purge was given, and oil and limewater poultices were applied to soften and remove the crusts. Two lays after her arrival, the patient was put upon purgative doses of the strong sulphur-water before breakfast, and diuretic doses of the mild sulphur-water during the day. As she was too weak for baths, I ordered warm mild sulphur-water to be used as a lotion morning and evening. In four days she was strong enough to go to the baths, and continued for a fortnight taking a mild sulphur-bath every alternate day, and the sulphur-waters.

At the end of the third week all signs of the impetiginoid tendency had disappeared, and the skin was rapidly becoming healthy. I then ordered her Kissingen-water as an aperient before breakfast, and small doses of the same water as a tonic during the day, also needle-baths on the alternate days. In a week her strength had increased so much, that I ordered her to have a sulphur-bath every day, to continue the Kissingen water as an aperient, but to change to the chloride of iron spa as a tonic. A fortuight later, the patient went home entirely recovered.

Mrs. J. had had slight attacks of eczema before, but never of the impetiginous form. The attack had lasted for ten weeks previously to her arrival in Harrogate, and had thoroughly resisted the ordinary methods of treatment.

On January 10th, my father received a letter from the Rev. E. G. $\mathrm{T}$., stating that his skin was very troublesome, and asking how soon he might venture to visit Harrogate. Before he could receive a reply, $\mathrm{Mr}$. T. arrived, and reported himself at once. $\mathrm{His}$ age was 37 . He had had four previous attacks, and when seen had eczema from heal to foot, partly squamous and partly weeping. From the knees downwards, we found, superadded to the eczema, a crop of purpuric spots, very numerous, with a distinct pustule filled with yellow pus in the centre of each. On the arms, from the elbows, and on the balls of the thumbs, were numerous scattered subcutaneous nodules, very lard, neither painful nor irritable, about the size of a millet-seed. There was no fever; the pulse and temperature were normal; the tongue coated. The patient slept badly, owing to the violent irritation of the eczema. 'The combination of the pustules with the purpura on the legs, and the hard millet-seed nodules with the eczema on the arms, was so unique, that the patient's history was very fully gone into. We learned that he had been living in a house adjoining an old brewery, built before the drainage-system of the town was completed, and never connected; it had been occupied by a care-taker for some years previously to his taking possession. Soon after he went into it, he and his friends perceived a smell in the kitchen and bath-room the sinks and closets were trapped, but the smell remained very bad at times. Mr. T. had three servants, and three friends living with him. The health-record of the six was as follows. The cook, aged 22, often had fainting fits, and contracted a troublesome cough, which lasted for months, and is not yet entirely shaken off. The housenaid, aged 19 was obliged to leave, and go into the infirmary for some female disorder, but had been well ever since leaving. The housekeeper had slight attacks of low fever. Friend No. 1 hail bad health all the time he lived in the house ; on going home, he had an attack of diphtheria. Friends No. 2 and 3 did not suffer. As regards Mr. T. himself, he had a bal attack of cczema, constantly woke with headache, and felt languid and tired. We put Mr. T. upon purgative loses of the sulphurwater in the molning, and the magnesia-water at midday; he was ordered a sulphur-bath every day. After very few baths, the purpuric pustules were gone, and the millet-seed nodules were less. In ten days the water was changed from sulphur to Kissingen, and from magnesia to chloride of iron; the purpura and remains of the millet-seed bodies rapidly disappeared, and, at the end of five weeks, the ecrema was also cured. In a letter to us, three weeks after leaving, the patient says he is perfectly well. 